\title{
Comprehensive catecholaminergic projectome analysis reveals single-neuron integration of zebrafish ascending and descending dopaminergic systems
}

Tuan Leng Tay', Olaf Ronneberger ${ }^{2,5}$, Soojin Ryu ${ }^{1, \dagger}$, Roland Nitschke $^{3,5} \&$ Wolfgang Driever ${ }^{1,3,4,5}$

Essential components of animal behaviour are modulated by dopaminergic (DA) and noradrenergic circuitry. In this study, we reveal at cellular resolution the complete set of projections ('projectome') of every single type of DA and noradrenergio neurons in the central nervous system of zebrafish larvae. The most extensive DA projections are established by posterior tubercular otp-dependent neurons, with individual somata integrating the ascending DA system, the descending diencephalospinal, as well as the endohypothalamic circuitry. These findings suggest a major role in the modulation of physiology and behaviour for otp-dependent DA neurons, which correlate with the mammalian A11 group. We further identified an endogenous subpallial DA system that not only provides most of the local DA projections, but also connects to the ventral diencephalon. The catecholaminergic projectome map provides a framework to understand the evolution and function of these neuromodulatory systems.

\footnotetext{
${ }^{1}$ Developmental Biology, Institute Biology I, Faculty of Biology, Albert-Ludwigs-University Freiburg, Hauptstrasse 1, Freiburg D-79104, Germany. ${ }^{2}$ Department of Computer Science, Albert-Ludwigs-University Freiburg, Georges-Koehler-Allee Geb 052, Freiburg D-79110, Germany. ${ }^{3}$ ZBSA Zentrum für Biosystemanalyse, Albert-Ludwigs-University Freiburg, Habsburgerstr. 49, Freiburg D-79104, Germany. ${ }^{4}$ Freiburg Institute for Advanced Studies, Albert-Ludwigs-University Freiburg, Albertstrasse 19, Freiburg D-79104, Germany. ${ }^{5}$ BIOSS_Center for Biological Signaling Studies, University of Freiburg, Albertstrasse 19, Freiburg 79104, Germany. †Present address: Max Planck Institute for Medical Research, Jahnstrasse 29, Heidelberg D-69120, Germany. Correspondence and requests for materials should be addressed to W.D. (email: driever@biologie.uni-freiburg.de).
} 
$\mathrm{D}$ istinct dopaminergic (DA) neuronal groups establish local and far-ranging projections to modulate circuits controlling many essential aspects of behaviour, cognition and physiology. Much attention has focused on the DA systems, given their clinical relevance in neurological and psychiatric disorders such as Parkinson's disease. Knowledge of the complete set of DA projections would improve the understanding of neural circuit function in the normal brain, the pharmacological treatment of diseased states and the requirements for neurorestorative therapies of degeneration. However, the complexity in the number of neurons and connections in the vertebrate brain has so far prohibited global approaches to comprehend 'connectomes'.

Studies in rodent, bird, amphibian and fish vertebrate models have revealed conserved and evolutionary dynamic aspects of catecholaminergic (CA) systems ${ }^{1,2}$, which in the central nervous system (CNS) consist of DA and noradrenergic (NA) neurons ${ }^{1}$. An advantage of zebrafish embryos and larvae as an experimental system is their translucent CNS, small enough to be imaged at cellular resolution in toto. Furthermore, a functional nervous system is established after only 4-5 days of embryonic development and enables zebrafish larvae to perform complex behaviours such as swimming and hunting ${ }^{3}$. Most of the analysis of zebrafish CA systems is based on the detection of Tyrosine hydroxylase (TH) protein or th transcript expression, which starts before the end of the first day of development ${ }^{4-7}$. A second paralogous th gene, th2, is expressed later than $t h$, and is predominantly observed in caudal hypothalamic DA neurons during early larval stages ${ }^{8-10}$. Zebrafish DA neurons form in the diencephalon and telencephalon, but not in the mesencephalon, wherein groups A8-A10 form in mammals ${ }^{4-6,8-10}$. However, ventral diencephalic DA neurons of the zebrafish posterior tuberculum that emanate ascending projections to the subpallium (SP) have been suggested to provide functions similar to the mammalian mesodiencephalic DA systems ${ }^{11,12}$. Mammalian DA groups are numbered in a caudal (A1) to rostral (A17) order ${ }^{1,2}$, and may be compared with zebrafish DA systems on the basis of anatomy and molecular markers (see Supplementary Note 1). Similar to A11 DA cells in mammals, zebrafish ventral diencephalic DA groups DC2 and DC4-6 are specified by the transcription factor Orthopedia (Otp) $)^{13-15}$. The other DA groups correspond to their mammalian anatomical counterparts in the hypothalamus (A12, A14-A15) and ventral thalamus (A13). Similar to mammals, NA cell groups are located in the locus coeruleus (LC), medulla oblongata (MO) and area postrema (AP) ${ }^{16-18}$.

Although CA axonal tracts in larval ${ }^{4,6,12,19}$ and adult $5,11,16,18,20$ zebrafish have been described, projections of individual neurons and their target areas have not been identified previously. In this study, we investigate the complete projections ("projectome ${ }^{21}$ ) of DA and NA systems in the larval zebrafish. To accomplish this goal, we tagged individual CA neurons by green fluorescent protein (GFP) expression using genetic mosaics and generated a large data set from which the projection behaviour of each type of th-expressing CA neuron in zebrafish larvae could be deduced. Our data revealed that A11-type DA neurons are the major far-projecting DA neurons in zebrafish, with single neurons projecting to telencephalon, diencephalon, hindbrain and spinal cord. Furthermore, we showed that a local subpallial DA system provides the major DA projections into the anatomical equivalent of the striatum in zebrafish. Our findings establish an anatomical framework for understanding the DA modulation of behaviour in zebrafish.

\section{Results}

Genetic labelling of single CA neurons and their processes. We introduced a Ras membrane-anchored EGFP (rasGFP) reporter at the ATG of the reading frame into a bacterial artificial chromosome (BAC) vector containing a $154 \mathrm{kbp}$ upstream region and most of the zebrafish tyrosine hydroxylase (th) gene. We chose th because at larval stages it is expressed in nearly all zebrafish
CA groups, whereas the paralogous th2 gene is expressed only in a small number of hypothalamic DA neurons ${ }^{8-10}$. Expression of th:rasGFP BAC is specific for th (Supplementary Fig. S1). th:rasGFP was injected into one-cell-stage embryos and efficiently labelled individual CA somata and their axons in genetically mosaic larvae. Systematic injections established a large data set of labelled neurons and enabled recording of axonal projection patterns for each population of CA neurons in zebrafish CNS at 4 days post fertilization (d.p.f.). At this stage, most CA neuronal clusters and tracts observed in the adult brain are established ${ }^{4,12}$. Diencephalic DA neuronal axons reach the spinal cord in as little as $12 \mathrm{~h}$ after onset of th expression ${ }^{6,12}$. Therefore, most DA axons are expected to have reached their larval stage targets at 4 d.p.f. Larvae $(n=408)$ coimmunostained with anti-GFP and anti-TH antibodies were analysed by confocal microscopy. Anti-GFP immunoreactive (ir) cell somata were identified as members of each of the previously defined DA neuronal groups on the basis of coexpression of $\mathrm{TH}$, thus revealing that th:rasGFP drives GFP expression in all known THir CA groups. A total of 310 larvae containing 670 GFP-labelled somata with distinct, non-intersecting projections contributed to the final data set used in this study (Supplementary Table S1).

We established an image analysis workflow to obtain a data set of single CA neuron projections and to identify and classify their projection behaviour (Fig. 1; Supplementary Methods, Supplementary Movie 1 and Supplementary Fig. S2). This imaging technique did not allow us to distinguish between axons and dendrites. Target area anatomical identities were confirmed from histological stains. To comprehensively analyse the CA projection network, we first dissected the characteristics of projections emanating from neurons of each CA group to include directionality (based on the body axes) and type (for example, arbourizing, branching, midline crossing, local; Supplementary Fig. S2). For further classification, major projections that are proximal to the cell soma and target the contralateral side were indicated as contralateral, whereas distal branches that originate from major ipsilateral projections but target contralaterally were documented as midline crossing processes. The second step was to visually trace all CA projections to their anatomical target areas (Fig. 2). Statistical analysis indicates that our data set is large enough to identify most ( $>80 \%$ ) of the projection behaviours and target areas.

Overview and classification of projections and target areas. Figure 2 summarizes projection patterns of all CA groups. Our further analysis structured the CA groups into five classes. First, NA systems comprise LC, MO vagal and AP neurons that share the main feature of far-ranging projections (Fig. 3), whereas NA neurons of the MO interfascicular zone predominantly connect locally (Supplementary Note 2 and Supplementary Fig. S3). The second class includes local as well as intermediate length projections of DA neurons: the larval DC1 group, which contains ventral thalamic and periventricular posterior tubercular DA neurons, and the pretectal DA group (Fig. 4; detailed in Supplementary Note 3). Class 3 DA groups project exclusively locally or to adjacent brain territories: hypothalamic anterior preoptic, preoptic, DC3 and DC7 groups, as well as olfactory and retinal DA groups (Fig. 4; Supplementary Note 4 and Fig. S4). In class 4, DA connections to distant brain regions appear to be predominantly formed by the otp-dependent diencephalic DA groups 2, 4, 5 and 6 (Figs 5-7). Finally, in the subpallium local DA neurons provide the predominant DA arbourization as well as descending projections to the hypothalamus (Fig. 8).

Complexity of projection patterns. Comparison of projection patterns shown by single neurons within each group led us to note that high individual neuron target variability exists. Taking DC 5 $(n=110)$ as an example, although the neurons predominantly project into the endohypothalamic tract (eht) and their descending axons terminate in the hindbrain or spinal cord, we identified different 
a

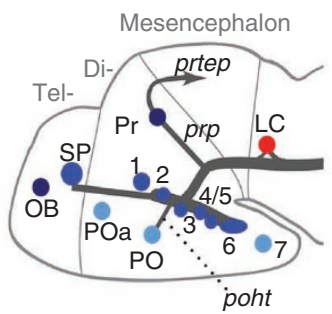

b
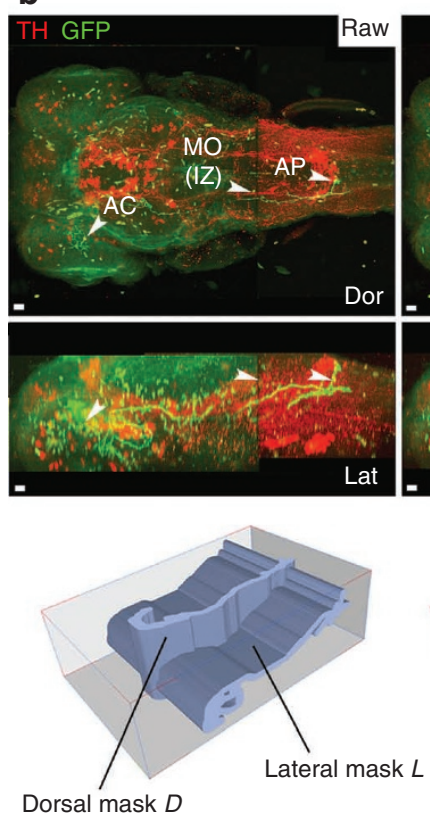

Dorsal mask $D$ poht
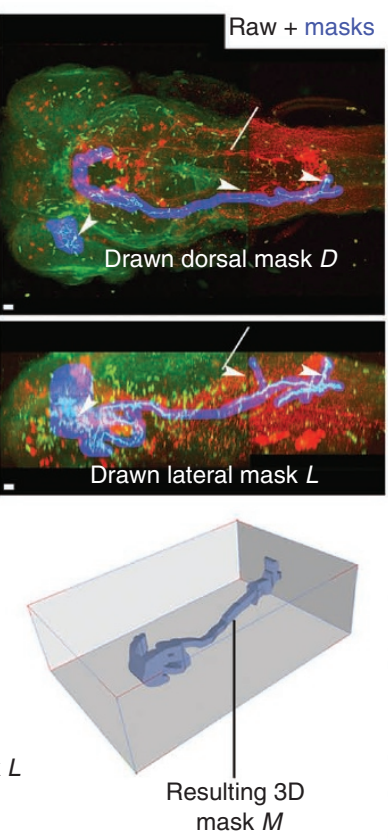

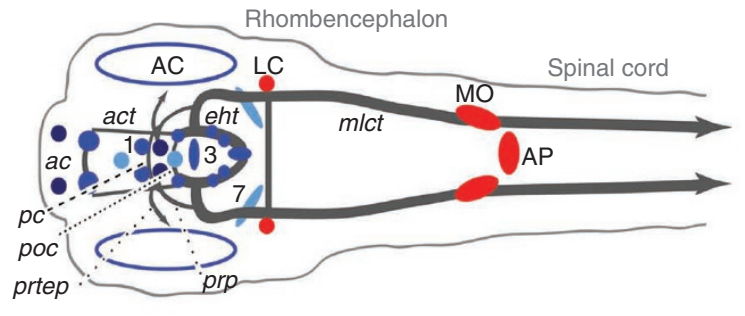

C

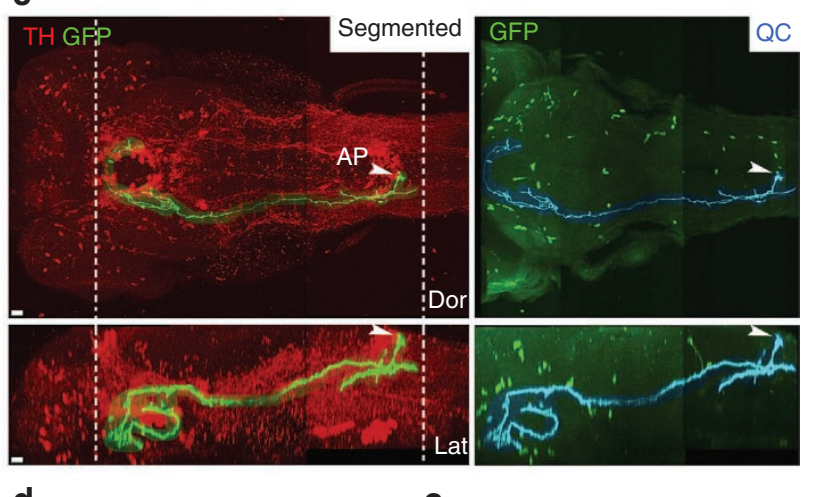

d

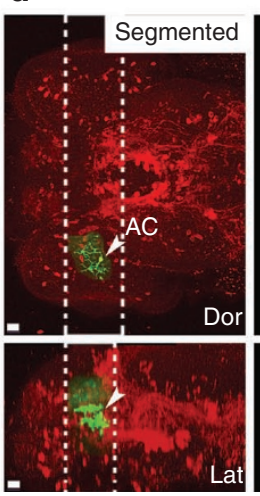

e

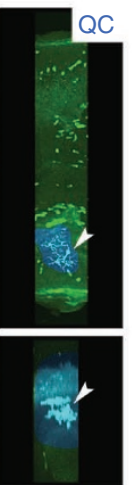

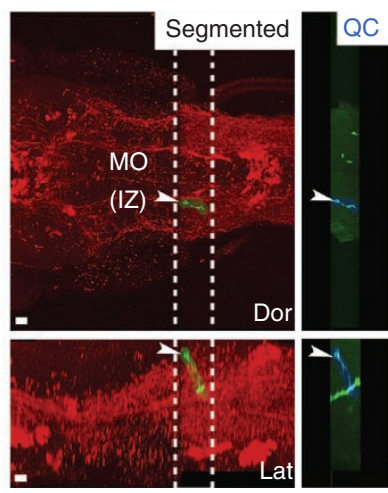

Figure 1 | Analysis of individual zebrafish larval catecholaminergic neurons and projections in the context of the intact brain. (a) Scheme of dopaminergic (DA; blue) and noradrenergic (NA; red) cell groups and catecholaminergic (CA) tracts (grey) in 4 d.p.f. zebrafish larvae ${ }^{12}$. Left: lateral view of forebrain and midbrain; right: dorsal view of CNS. Dark blue indicates dorsal-most and light blue ventral-most DA groups. Diencephalic DA groups: (DC1) ventral thalamus and periventricular posterior tuberculum; (DC2, DC4) large neurons in posterior tuberculum; (DC5, DC6) medium-sized neurons in posterior tuberculum and hypothalamus; (DC3) medial hypothalamus; (DC7) caudal hypothalamus; $\mathrm{PO}$, preoptic region; POa, anterior preoptic region; Pr, dorsal pretectum; AC, retinal amacrine cells. Telencephalic DA groups: SP, subpallium; OB, olfactory bulb. NA groups: LC, locus coeruleus; MO, medulla oblongata interfascicular zone and vagal area and AP, area postrema. ac, anterior commissure; act, anterior CA tract; eht, endohypothalamic tract; mlct, medial longitudinal CA tract; pc, posterior commissure; poc, postoptic commissure; poht, preopticohypothalamic tract; prp, pretectal projections; prtep, pretectotectal projections. (b-e) In silico separation of individual neurons (arrowheads in b) and their projections from whole-mount confocal image stack raw data. (b) Unprocessed maximum intensity projection (MIP) of a 4 d.p.f. larval brain. Blue masks $D$ and $L$ were manually drawn on the MIPs using ImageJ to separate soma and projections, based on visual discrimination. Bottom-most panel shows an example for computation of $3 D$ segmentation mask $M$ for a single neuron in the AP, based on the drawn masks $D$ and $L$. The resulting $3 D$ mask $M$ was applied to the green channel to isolate the neuron and its projections. (c) Visualization of segmented AP neuron shown in (b) with projections. Quality control (QC; cyan) was carried out in 3D space within the region of interest (between dotted lines) to ensure the masks did not omit any GFP-labelled features belonging to the particular neuron. (d, e) Visualization of segmented individual soma and projections of GFP-labelled (d) amacrine cell (arrowheads) and (e) NA neuron in the MO interfasicular zone (IZ; arrowheads). See Supplementary Movie 1. Anterior at left, dorsal at top; anti-TH (red), anti-GFP (green); Dor, dorsal view; Lat, lateral view. Scale bars, $20 \mu \mathrm{m}$.

combinations of target choice by processes arising from individual somata. These variations may be qualitatively assessed from the selected examples in Supplementary Fig. S5. A detailed analysis of categories of projection behaviours is found in Supplementary Fig. S6. We emphasize this observation to highlight the complexity of the CA systems even though further investigation into this aspect is not within the scope of this study. To assess projection behaviours typical for a specific neuronal group, we conducted Fisher's exact test to mark projection behaviours typical for most neurons of each group (Supplementary Fig. S7).

Hindbrain NA neurons widely project throughout the CNS. The LC, MO vagal area (VA) and AP NA groups innervate a broad range of target regions extending from the forebrain to the spinal cord. The typical projection topology of individual somata starts with a single major axon leaving the soma, generating numerous subsequent 


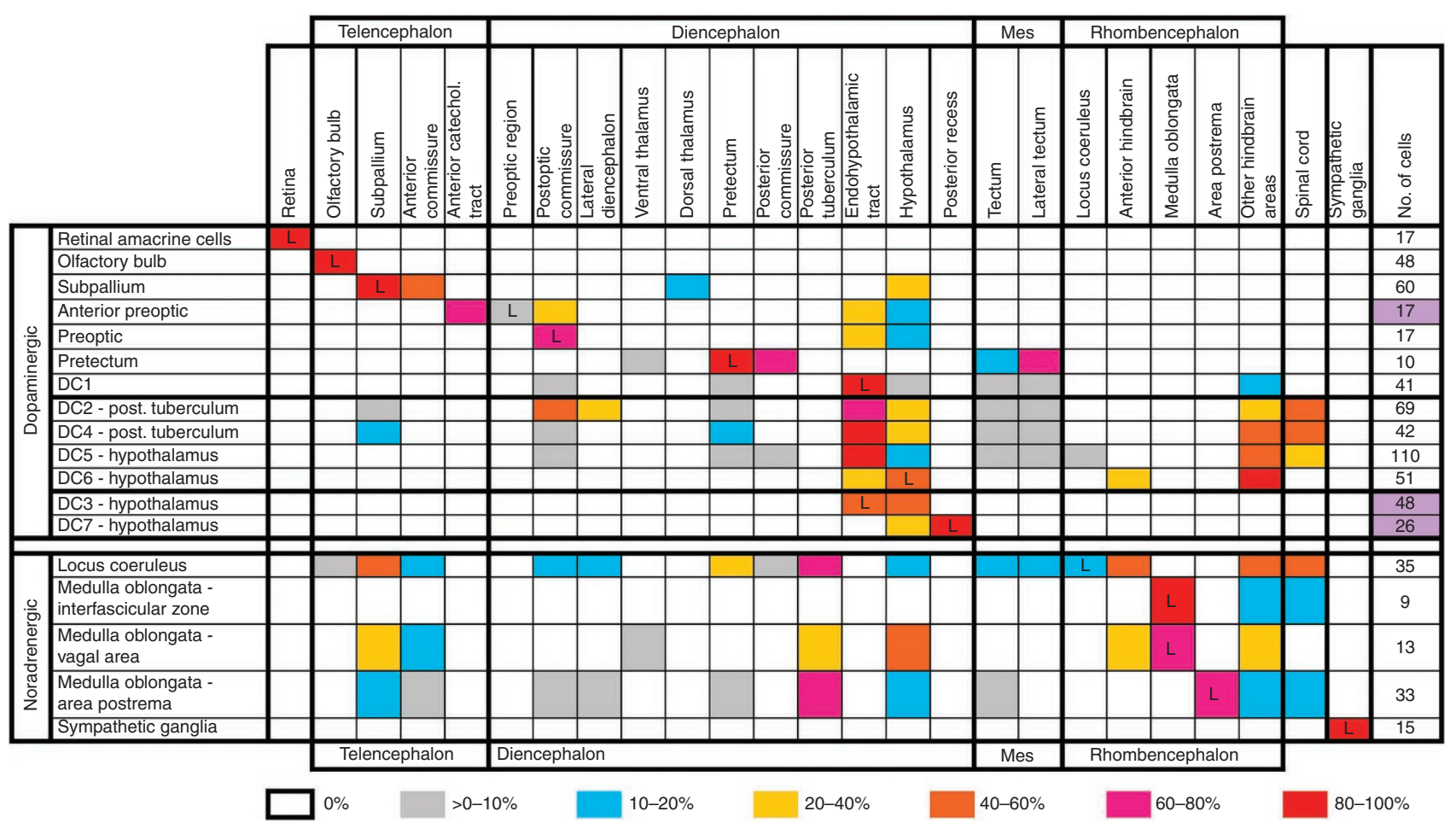

Figure $\mathbf{2}$ | Target areas of catecholaminergic projections. The target areas (columns) of projections of each catecholaminergic (CA) neuronal group (rows) are indicated quantitatively by the colour code representing the percentage of total neurons per analysed group that project into the area. The total number of neurons analysed is shown in the furthest right column. For CA groups in which individual somata extend projections to multiple target areas, the percentages add up to more than 100. Lavender indicates the neuronal group is liquor contacting. Projections pass through the commissures with the exception of a few cases in which some processes were noted to terminate there: anterior commissure as target of locus coeruleus and area postrema noradrenergic neurons; postoptic commissure as target of anterior preoptic, preoptic, DC1, 2, 4 and 5 dopaminergic neurons. ' $L$ ' (for local projection) denotes the termination of neuronal processes within the region in which the soma is located. DC1 includes populations located in the ventral thalamus and periventricular posterior tuberculum.

branches in up to six different brain territories (Figs 2 and 3; Supplementary Fig. S2). For LCNA neurons, this often includes ipsilateral and contralateral areas (14 of 35 somata; Fig. 3a; Supplementary Movie 2). LC neurons predominantly emanate ascending projections as far anterior as the SP and olfactory bulb, as well as to the posterior tuberculum and hypothalamus. Telencephalic targets of the LC that were not reflected in Figure 2 include the pallium $(n=3)$ and the pallialsubpallial border area $(n=3)$. In addition to forming local processes, LC NA neurons also send descending projections down the spinal cord. Of the LC-derived projections that target the anterior hindbrain (Fig. 2), 35\% terminated in the cerebellum. The main NA contributions to the pretectum (11 of 13 NA projections) and tectum (12 of 13 NA projections) were observed to originate from the LC (Fig. 2).

For analysis, we subdivided MO into the interfascicular zone, $\mathrm{VA}$ and $\mathrm{AP}^{18}$. The majority of somata from the VA (Fig. 3b) and AP (Fig. 3c; Supplementary Movie 3) were observed to send a farreaching axon and multiple processes into the telencephalon and diencephalon (41 of $46 \mathrm{MO}$ somata), in addition to strong local projections within the MO (Fig. 2). Approximately $69 \%$ of these MO-VA neurons formed forebrain projections to the SP, posterior tuberculum or hypothalamus. Similarly, most (28 of 33) of the examined AP NA neurons were found to emanate long ascending axons from an individual soma that target either both the telencephalon and different ventral diencephalic regions, or only one of these two target areas. They also arbourize into or sparsely innervate the AP (Fig. 3c). The remaining AP NA neurons projected only within the hindbrain (5 of 33). Furthermore, for $<10 \%$ of the cases we noted minor NA branched processes from the MO that contribute to spinal cord projections (Supplementary Fig. S2).
Projections of Otp-dependent DA neurons. Within the larval DA system, DC2, DC4, DC5 and DC6 are major contributors to the eht (201 of 280 DA processes to eht) and DA tracts to the hindbrain and spinal cord (237of 243 DA descending projections; Figs 2, 5 and 6). Most of these neurons develop complex branching patterns, often contributing contralateral projections in the hindbrain (49\%; Supplementary Fig. S2). Minor processes originating from DC2, DC4 and DC5 target the pretectal and anterior tectal areas (Fig. 2). A high proportion of individual neurons from these four clusters project locally within the ventral diencephalon (that is, local processes contribute to poc, eht and caudal hypothalamus), as well as to the hindbrain and/or spinal cord. However, the distinct Otp-dependent clusters behave differently with respect to the relative contribution to these target areas. Both local and descending projections were identified for $78 \%$ of all examined DC2 neurons, $90 \%$ of DC4, $64 \%$ of DC5 and 49\% of DC6 (Fig. 7; Supplementary Figs S2 and S6).

These findings prompted us to analyse whether any of the four Otp-specified, but anatomically distinct, DA groups exhibit specialized projection behaviours. Statistical clustering analysis revealed that, overall, descending projections are typical for Otp-dependent neurons (Supplementary Fig. S7). However, the neuronal groups differ in the caudal extent of their projections. Significant numbers of neurons documented for DC2 (45\%), DC4 (57\%) and DC5 (29\%) contribute to the spinal cord, whereas those of DC5 (63\%) and DC6 (86\%) preferentially ended within the rhombencephalon. The DC5 group targeted the LC significantly more often than other DC groups. In contrast to other otp-expressing groups, the most anterior group, $\mathrm{DC} 2$, more frequently projects ventrally into the preoptic region and laterally into the lateral diencephalon. This suggests that either 

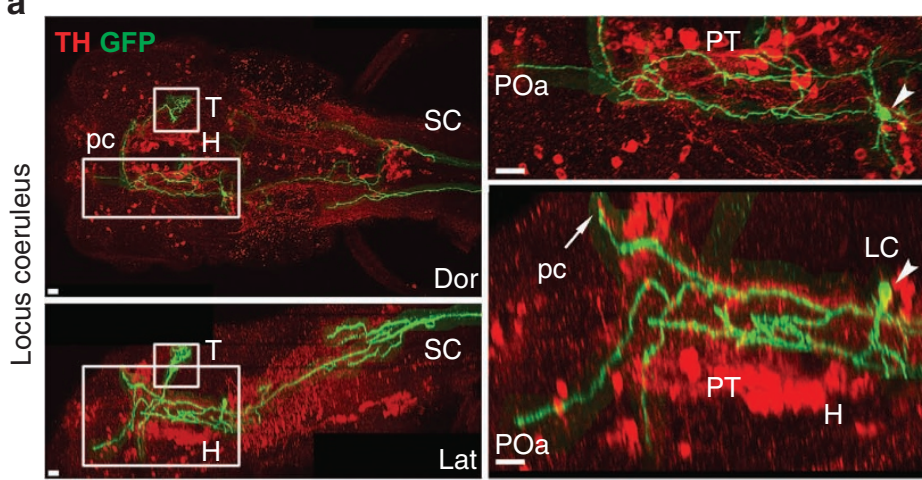

b

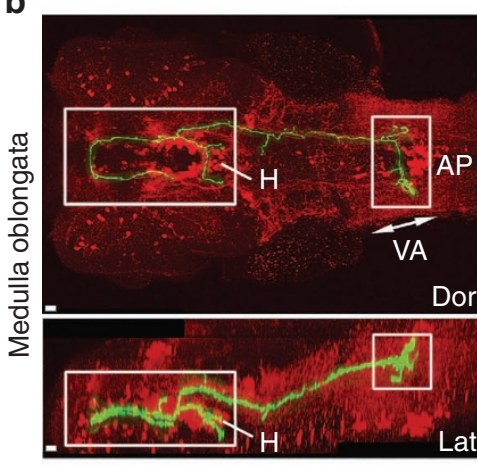

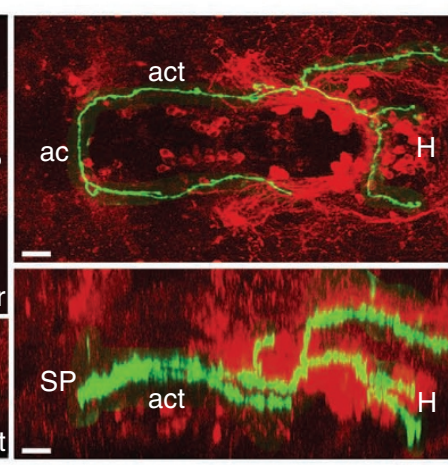

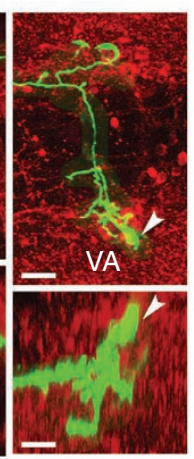

C
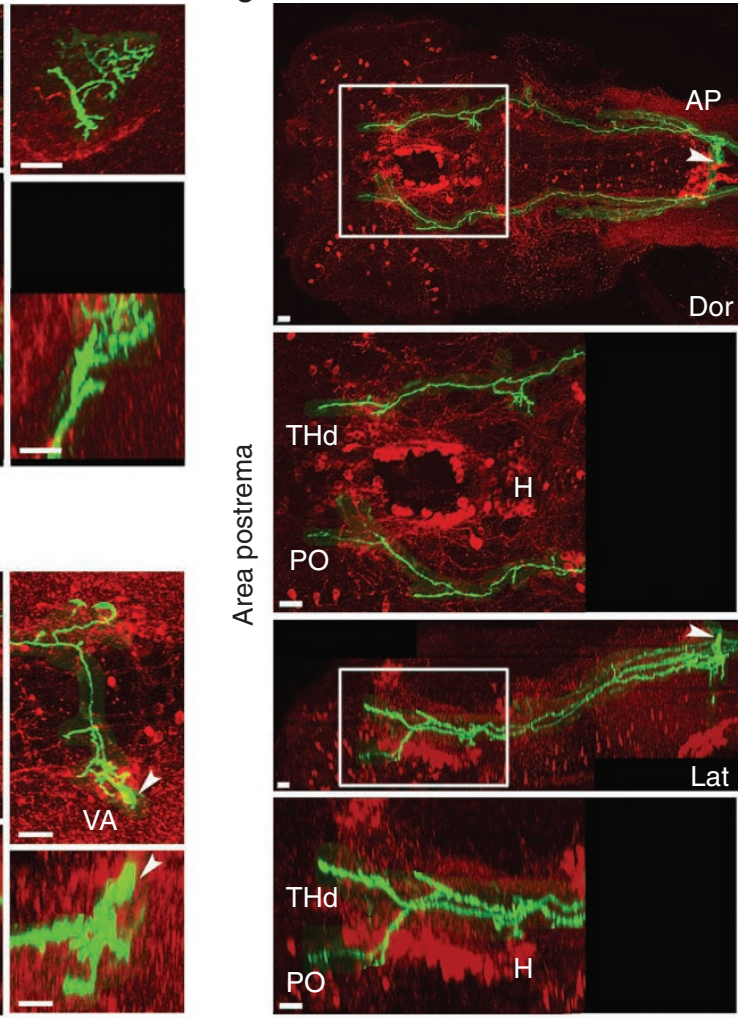

d
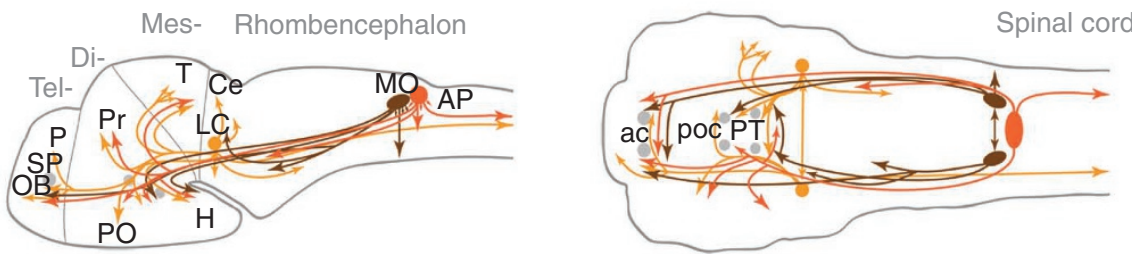

- Locus coeruleus

- Medulla oblongata (vagal area)

- Area postrema

Figure 3 | Long-range projections of hindbrain noradrenergic neurons. (a-c) Z projections of confocal stacks in dorsal (Dor) and lateral (Lat) views. (a) A locus coeruleus noradrenergic (NA) neuron (arrowheads) projects into multiple regions of the brain including posterior tuberculum (PT), anterior preoptic region ( $\mathrm{POa}$ ) and the spinal cord (SC) via a descending branched axon. A projection from the soma ascends and crosses the midline via the posterior commissure ( $\mathrm{pc}$; arrow in middle bottom panel), and forms an arbour of fine processes within the tectum ( $\mathrm{T}$; magnified in rightmost panels). Middle and right panels are magnification of boxes in leftmost panels. See Supplementary Movie 2. H, hypothalamus. (b) A medulla oblongata vagal area (VA) NA neuron (arrowheads) sends a far-reaching axon into the tel- and diencephalon. The long axon forms branches within the posterior tuberculum to innervate the hypothalamus as well as the contralateral side via the anterior catecholaminergic tract (act) and the anterior commissure (ac). A short, branched process (top right panel) appears to target the rhombencephalon. Local fibres emanate from the proximal part of the axon. Middle and right panels are magnification of boxes in left panels. (c) A single area postrema (AP) NA soma (arrowheads) sends long ascending axons terminating in contra- and ipsilateral sides of the diencephalon. The right projection ends in the dorsal thalamus (THd), whereas the left innervates the preoptic region (PO; magnified in below dor and lat panels). See Supplementary Movie 3. Anterior at left, dorsal at top; 4 d.p.f. larvae; anti-TH (red), anti-GFP (green). Scale bars, $20 \mu \mathrm{m}$. (d) Schematic overview of NA projections in 4 d.p.f. zebrafish larvae. Projection pathways (indicated by lines) and target areas (indicated by arrowheads) are depicted in lateral (left) and dorsal (right) views. Short arrows (see MO and AP) denote formation of local arbours or processes. For visual clarity in dorsal views, lines and arrows are not depicted left-right reciprocally. Our data indicate no asymmetry. Ce, cerebellum; $\mathrm{OB}$, olfactory bulb; $\mathrm{P}$, pallium; poc, postoptic commissure; Pr, pretectum; SP, subpallium.

unknown mechanisms generate subtypes among Otp-specified DA neurons, or different signalling environments of the anatomically distinct Otp groups direct different projection behaviours.

Ascending DA projections. We analysed ascending DA projections from the ventral diencephalon to the telencephalon. Only the more rostral Otp-dependent DC2 and DC4 neurons develop such projections. Among the $111 \mathrm{DC} 2 / 4 \mathrm{DA}$ neurons analysed, ten somata (9\%) sent an ascending projection to the SP (Figs 2, 6 and 7; Supplementary Fig. S2). Given that there are on average only 14.3 $( \pm 2.02)$ DC2/4 DA neurons in a 4-d.p.f. brain, these data reveal that there are only one or two DC2/4 neurons with ascending projections per larvae (Supplementary Table S2). In addition, these large, pearshaped unipolar cells ${ }^{4}$ have fine local bifurcations and send processes to hypothalamic domains, as well as far-descending axons that target the hindbrain and may traverse the spinal cord (Figs 6 and 7; Supplementary Movie 4). Such projection characteristics are also typically observed for the majority of DC2, 4 and 5 neurons that do not send ascending projections (Fig. 7; Supplementary Fig. S6). Our counts of DC2/4 somata (left: $7.5 \pm 1.6$, right: $6.8 \pm 0.9$; Supplementary Table S2) are similar to those reported for the adult zebrafish brain ${ }^{22}$. This confirms that the number of large, pear-shaped 

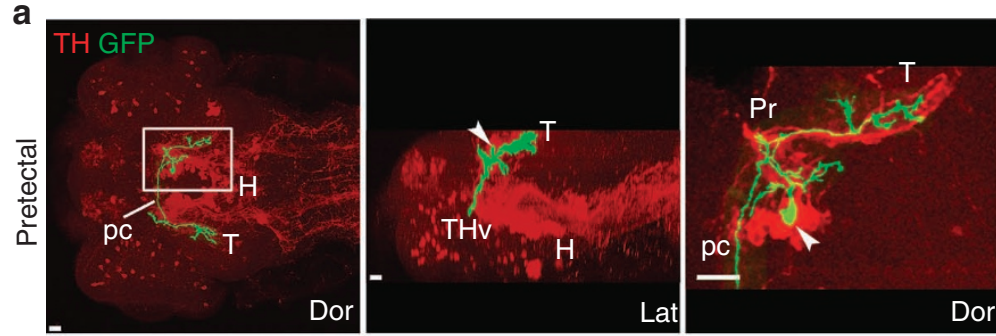

b
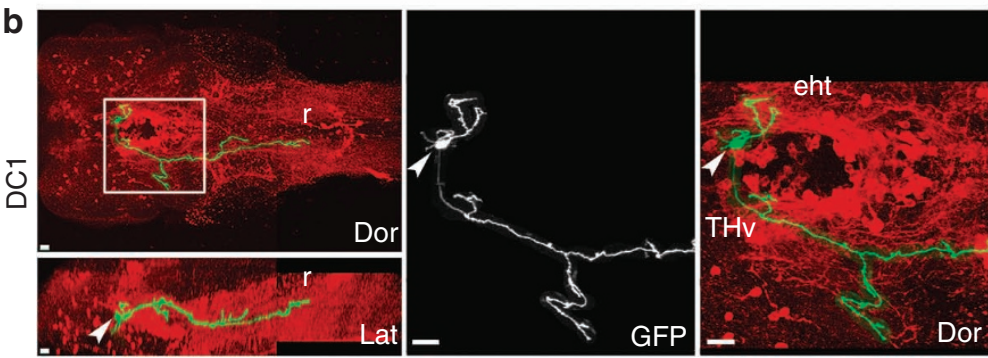

f
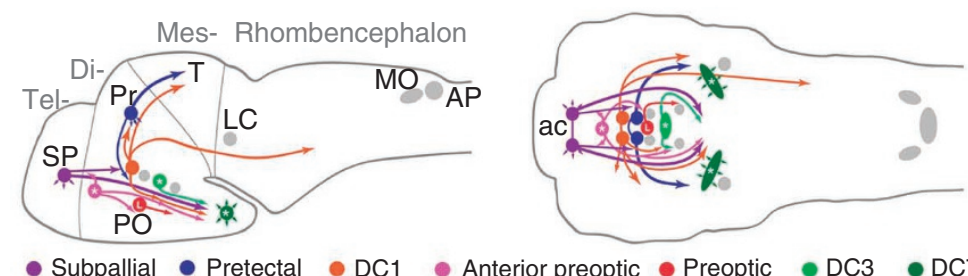

Anterior preoptic Preoptic - DC3 - DC7
C
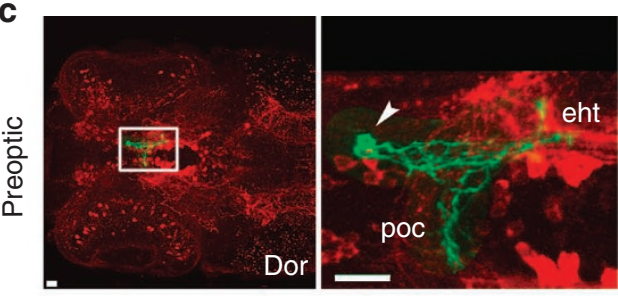

d
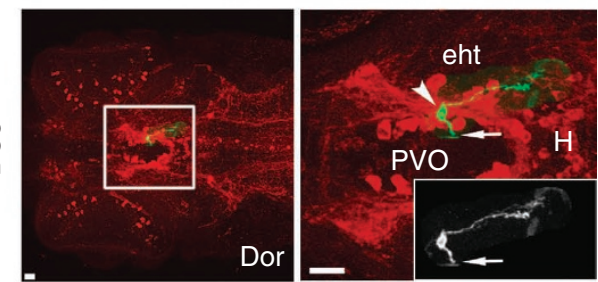

e
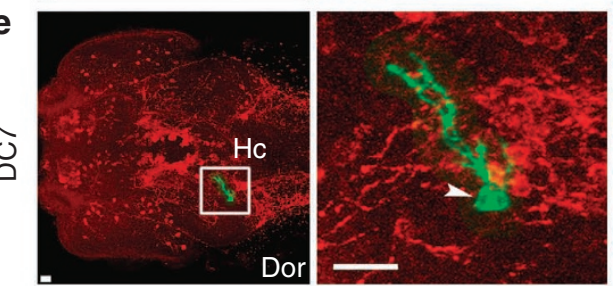

Figure 4 | Dopaminergic groups with characteristic short-range or local projection behaviours. (a-e) Z projections of confocal stacks in dorsal (Dor) and lateral (Lat) views. (a) A pretectal dopaminergic (DA) neuron (arrowheads) forms a local arbour within the pretectum (Pr) and projects into the ipsilateral tectum ( $\mathrm{T}$; magnified in right panel) and across the posterior commissure (pc) to the contralateral tectum (left panel). $\mathrm{H}$, hypothalamus. (b) A diencephalic cluster (DC) 1 neuron (arrowheads) sends short proximal processes within the ventral thalamus (THv), into the endohypothalamic tract (eht) (magnified in middle and rightmost panels), and descending projections into the rhombencephalon ( $r$ ). (c) A preoptic DA neuron (arrowhead) projects into the postoptic commissure (poc) and eht (magnified in right panel). (d) A DC3 neuron (arrowhead) located in the paraventricular organ (PVO) is liquor contacting (arrows pointing to end-foot) and contributes to the eht and hypothalamus (magnified in right panel; inset shows GFP signal). (e) Liquor-contacting DC7 soma (arrowhead) within the posterior recess possesses sparse processes (magnified in right panel) that target locally in the caudal hypothalamus ( $\mathrm{Hc}$ ). Anterior at left, dorsal at top; 4 d.p.f. larvae; anti-TH (red), anti-GFP (green, white in panels with black contrast). Scale bars,

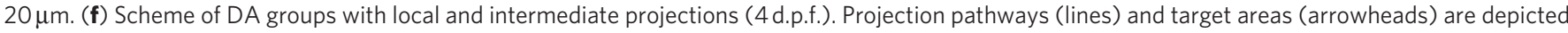
in lateral (left) and dorsal (right) views. Short arrows and ' $L$ ' denote local arbours or processes. An asterisk (*) marks liquor-contacting DA groups. For visual clarity, lines and arrows are not depicted left-right reciprocally in dorsal views. Our data indicate no asymmetry. ac, anterior commissure; AP, area postrema; LC, locus coeruleus; and MO, medulla oblongata.

DC2/4 DA neurons does not increase from larval to adult stages. Analysing laterality, we found no preferential DA ascending projections from either side of the posterior tuberculum (one-tailed paired $t$-test $>0.278$, Supplementary Table S3).

Prominent local projections of subpallial DA neurons. As the majority of telencephalic DA fibres do not originate from the ventral diencephalon, we examined the origin of the dense THir fibres within the SP. Among 60 GFP-labelled subpallial DA neurons analysed, 55 (91.7\%) were found to radiate dense local arbours within their territory. The prominent arbourization appears to cover the whole SP, whereas DA axons appeared not to project deep into the pallium. No projection to olfactory telencephalic territories was detected. Of the 60 somata, 25 sent a single contralateral process into the region of the anterior commissure. A substantial fraction of subpallial DA neurons also project a descending axon into the dorsal thalamic (12 of 60) or hypothalamic (18 of 60) regions, often in combination with local subpallial arbours and contralateral projections (Figs 4f and 8; Supplementary Fig. S8, Supplementary Movie 5). Given the late differentiation of subpallial DA neurons, the dorsal thalamus may also be an intermediate target at 4 d.p.f. of hypothalamus-bound descending projections. To determine whether left-right asymmetry exists in the diencephalon-bound descending axons, the number of subpallial somata and projections on both sides of the telencephalon was counted. There are an average of $10.7( \pm 1.9)$ and 10.3 $( \pm 2.5)$ neurons on the left and right SP, respectively (two-tailed paired $t$-test $>0.379$, Supplementary Table S4). We observed 14 descending processes on the left and 16 on the right SP (one-tailed paired $t$-test $>0.352$, Supplementary Table S5). We therefore conclude that no chirality in the number of telencephalic descending connections to the diencephalon was observed. Semiquantitative abstraction of our tally of descending projections from subpallial DA neurons (30 of 60) predicts that in a 4 d.p.f. larval brain containing $21( \pm 3.9)$ of these somata, $10-11$ neurons (50\%) are likely to project to the diencephalon, therefore forming the major DA connections between the rostral and caudal forebrain. Thus, in quantitative terms, the predominant DA connection between the SP and ventral diencephalon is not ascending, but descending.

\section{Discussion}

Understanding the projection behaviour of CA neurons is essential to evaluate their modulatory input into circuits that control behaviours ranging from motor initiation to addiction. We used genetically mosaic zebrafish to systematically label each type of CA neuron, and 
a
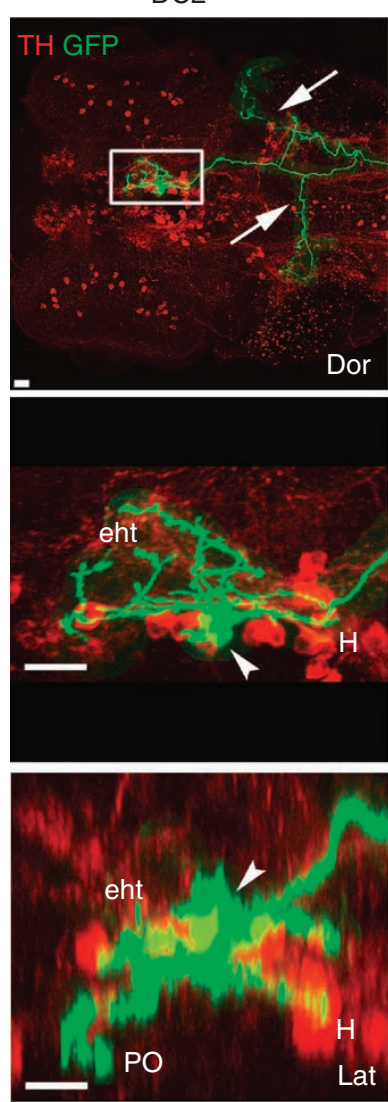

b
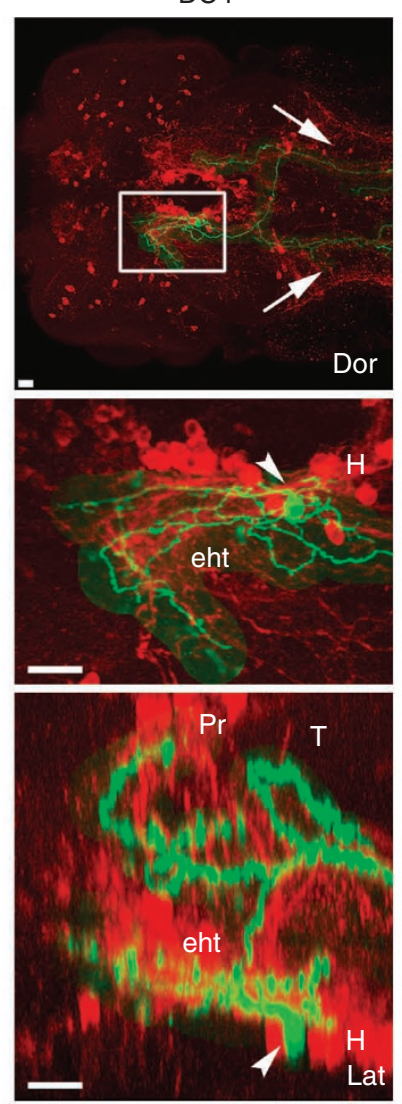

C
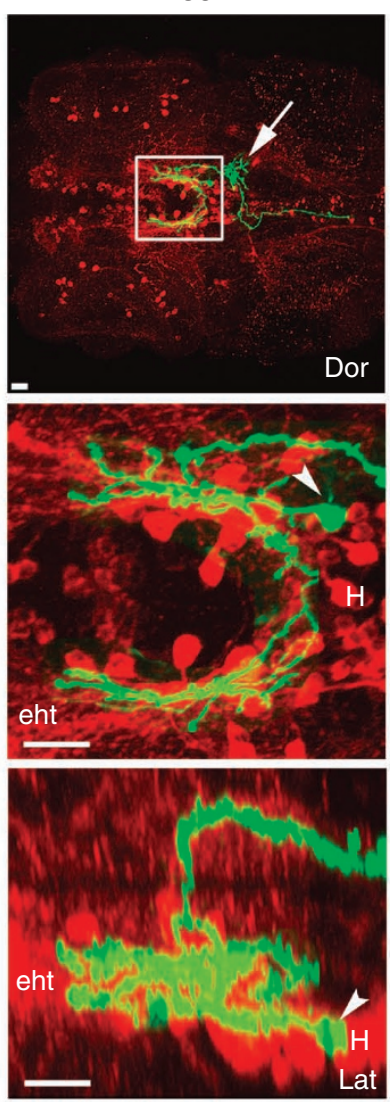

d
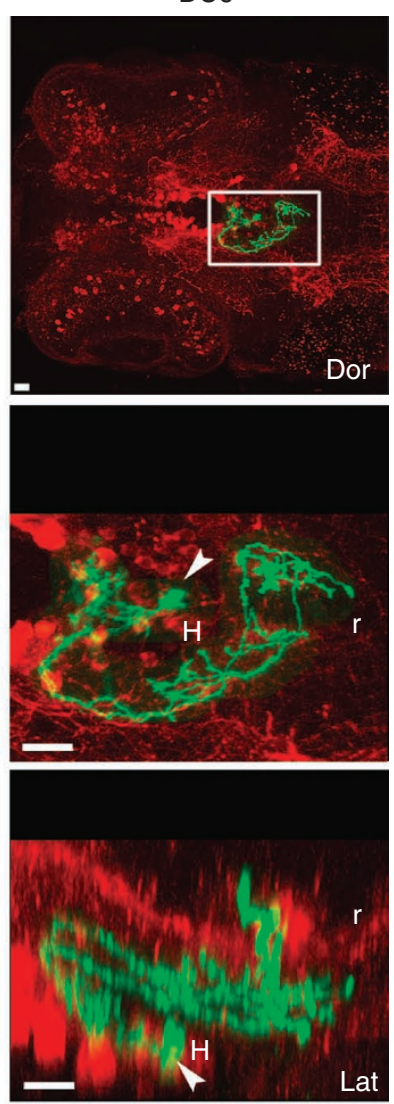

e
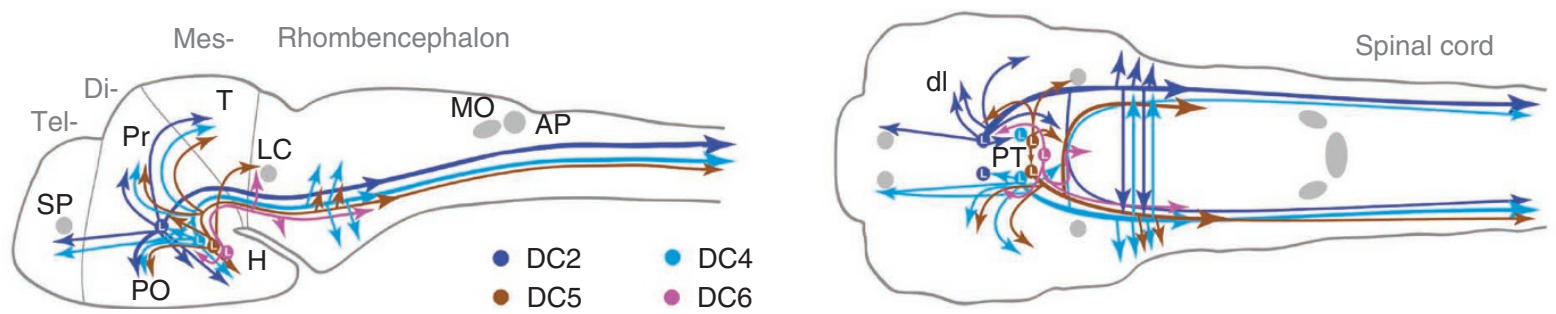

Figure 5 | Projection behaviours of otp-dependent dopaminergic diencephalic clusters. (a-d) Z projections of confocal stacks in dorsal (Dor) and lateral (Lat) views. Lateral views correspond to magnified regions (middle panels). (a) A DC2 neuron (arrowheads) contributes to the endohypothalamic tract (eht), forms local arbours and ventral processes into preoptic region (PO) and hypothalamus (H) (magnified in middle panel). A long axon descends into the hindbrain with branches (arrows) across the midline, and into the spinal cord (not shown). (b) A DC4 soma (arrowheads) with short processes contributing to the eht and connecting within the hypothalamus (magnified in middle panel), a dorsal branch extending towards the lateral pretectum (Pr) and tectum (T) (bottom panel), and long descending axons (arrows) into left and right hindbrain. (c) A DC5 soma (arrowheads) with ipsilateral and contralateral contributions to the eht (magnified in middle panel). From the ipsilateral eht, a branch (arrow) extends dorsoposteriorly into the medial longitudinal catecholaminergic tract to the hindbrain. (d) A DC6 axon (arrowheads) arbourizes extensively in the hypothalamus (magnified in middle panel) and projects towards the caudal midbrain and anterior hindbrain (bottom panel). r, rhombencephalon. Anterior at left, dorsal at top; 4 d.p.f. larvae; anti-TH (red), anti-GFP (green). Scale bars, $20 \mu \mathrm{m}$. (e) Schematic overview of DC2, DC4, DC5, and DC6 projection pathways (lines) and target areas (arrowheads) in lateral (left) and dorsal (right) views. 'L' denotes local arbours or processes. For visual clarity, lines and arrows are not depicted left-right reciprocally in dorsal views. Our data indicate no asymmetry. AP, area postrema; dl, lateral diencephalon; LC, locus coeruleus; MO, medulla oblongata; PT, posterior tuberculum; SP, subpallium.

to track their complete projections in three-dimensional (3D) space. This work generated the first complete view of the projection patterns that originate from CA somata in a larval vertebrate, providing a close representation of the CA neurotransmitter class-specific 'projectome' ${ }^{21}$. There are two inherent limitations in this study: first, our focus on the larval stage at 4 d.p.f. may not reveal aspects of the CA systems that mature later, including potential additional axonal tracts. However, important aspects of the larval DA systems resemble those found in the adult brain: 4 d.p.f. larvae have a diverse behavioural repertoire ${ }^{3}$, and the number of neurons in some CA clusters is highly similar (LC NA neurons ${ }^{17}$ ) or is in the same order of magnitude (Otp-dependent DC2 and DC4 DA neurons ${ }^{9,20,22}$ ) as in adults. Second, projections of th2-expressing neurons were not analysed, as our BAC and anti-TH antibody are specific for th and TH. However, several domains of th 2 expression overlap with those of THir neurons in the diencephalon ${ }^{9,10}$, and most th2 DA neurons are caudal hypothalamic, which may likely possess mainly local projections similar to DC7 in zebrafish and A12 in mammals. 

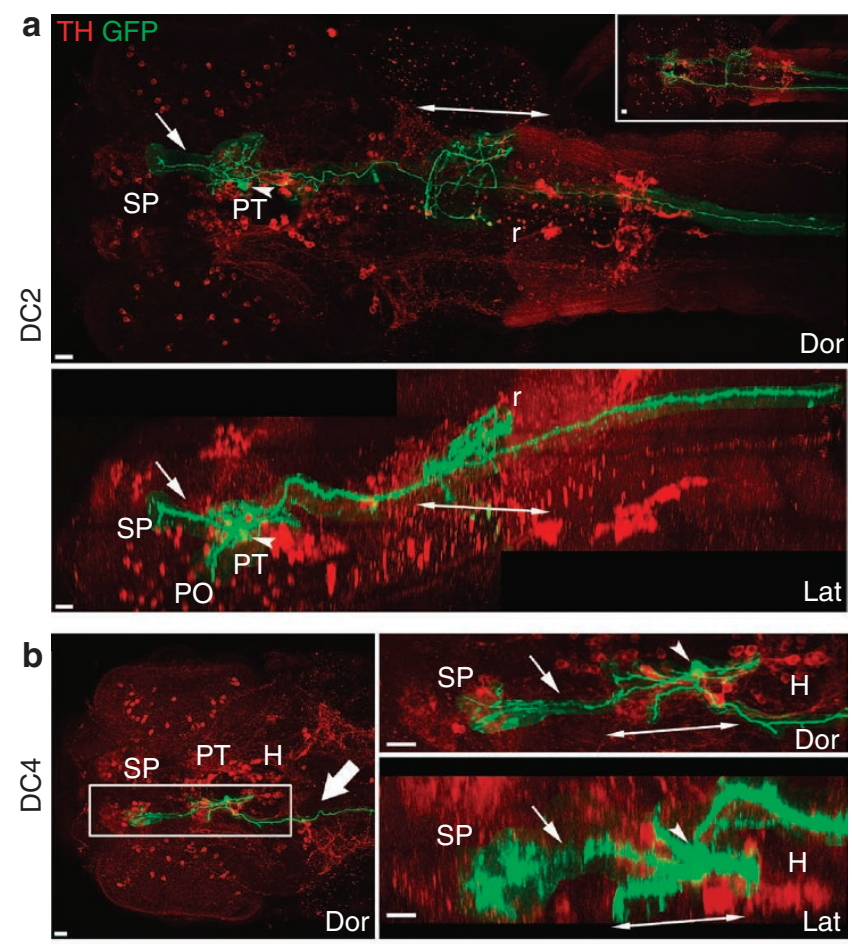

C

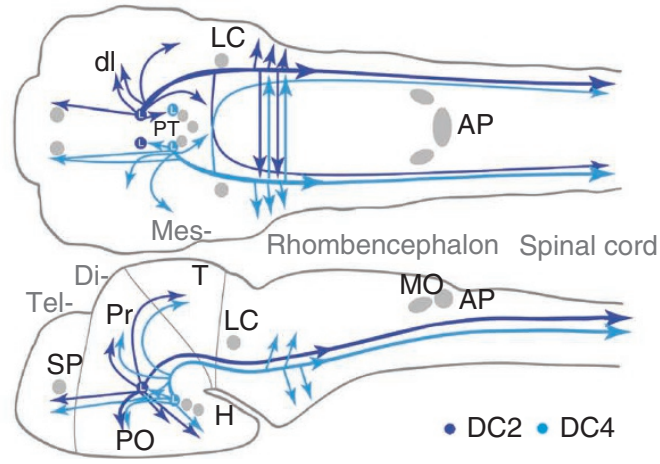

Figure 6 | Individual somata of DC2 and DC4 emanate both ascending and descending projections. $(\mathbf{a}, \mathbf{b}) \mathrm{Z}$ projections of confocal stacks in dorsal (Dor) and lateral (Lat) views. (a) A DC2 neuron (arrowheads) within the posterior tuberculum (PT) projects both an ascending axon (single arrows) to the subpallium (SP) and a descending axon into the spinal cord. This neuron also forms a local arbour in the PT and ventral processes targeting the preoptic region (PO). Branches derived from the main descending axon were observed in the rhombencephalon ( $r$ ), however, this region (double arrows) could not be clearly segmented because of the presence of a second GFP-labelled DC2 cell in the left PT, which contributed a midline-crossing branch into this area (inset). See Supplementary Movie 4. (b) A DC4 neuron (arrowheads) in the PT sends long ascending (single arrows) as well as descending (broad arrow) projections. Local processes in the PT and hypothalamus $(\mathrm{H})$ also originate from this DC4 soma (double arrows). Its ascending projection arbourizes within the SP (magnified in the right panels). Anterior at left, dorsal at top; 4 d.p.f. larvae; anti-TH (red), anti-GFP (green). Scale bars, $20 \mu \mathrm{m}$. (c) Schematic overview of DC2 and DC4 projection pathways (lines) and target areas (arrowheads). Dorsal (top) and lateral (bottom) views. 'L' denotes local arbours or processes. DC2 and DC4 neurons contribute to wide-ranging ipsilateral and contralateral projections into all major brain subdivisions. For visual clarity, lines and arrows are not depicted left-right reciprocally in dorsal views. Our data indicate no asymmetry. AP, area postrema; dl, lateral diencephalon; LC, locus coeruleus; MO, medulla oblongata; Pr, pretectum; T, tectum.

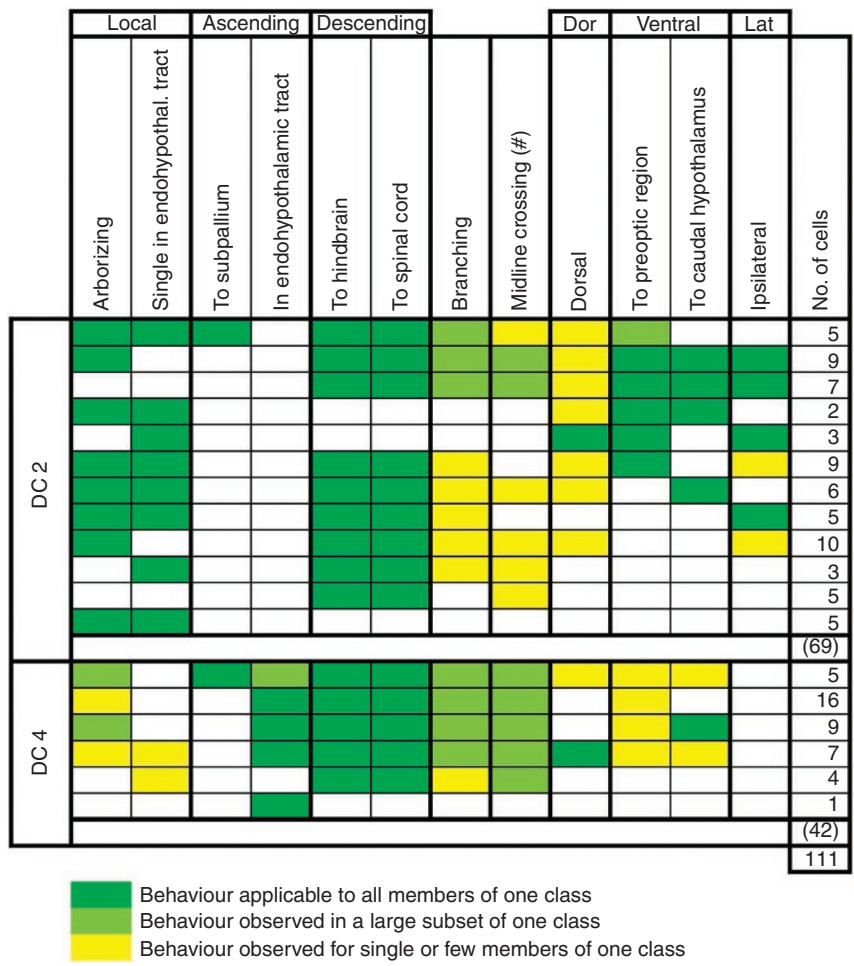

Figure 7 | Diversity of projection behaviour subtypes of individual DC2 and DC4 neurons. DA neurons of one anatomical group express high diversity of projection behaviours within defined repertoires. DC2 (69 somata) and DC4 (42 somata) neurons were analysed and shown to connect to tel-, di-, mes- and rhombencephalon. Individual somata usually connect through the endohypothalamic tract and send different combinations of projections to the subpallium, lateral forebrain, lateral tectum, hindbrain and/or spinal cord. DA innervation in the preoptic region and lateral forebrain primarily derives from DC2 cells. Individual somata were classified on the basis of their major projections (green), but smaller deviations in branching pattern and targets were allowed (yellow and light green). The rightmost column indicates the number of cells observed for each specific pattern (rows), and in parentheses the total number of cells analysed for DC2 and DC4. Dor, dorsal projections; lat, lateral projections; single in endohypothalamic tract indicates contribution of single axon to tract; (\#) ipsilateral distal branch that crosses the midline to target the contralateral side.

Our analyses of projection paths and target areas identified projection behaviours typical for each CA neuronal group. However, within the repertoire of projections characteristic for each group, individual neurons exhibited high variability in their projection paths and targets. The observed diversity may represent true additional genetically defined subtypes. Alternatively, projections formed by neurons of one subtype may depend on local guidance cues and may have stochastic aspects. Diversity in wiring diagrams of a single neuron type has previously been shown for vertebrate motor neuron connectomes ${ }^{23}$, in contrast to the more stereotyped wiring in invertebrates ${ }^{24}$.

Our study sheds light on evolutionarily conserved and dynamic aspects of CA systems in vertebrates. Compared with mammals ${ }^{25}$, our data revealed that all significant aspects of zebrafish NA projections from the LC and MO appear to be conserved. Although projections from caudal rhombencephalic NA groups into the forebrain have not been reported for adult cypriniform fish ${ }^{11,26,27}$, other far-projecting brainstem transmitter systems have been described in perciform fish ${ }^{28}$. Our findings are consistent with a fundamental modulatory role of LC NA connectivity in global behavioural patterns, ranging from basic motor behaviours and stress responses to 
a

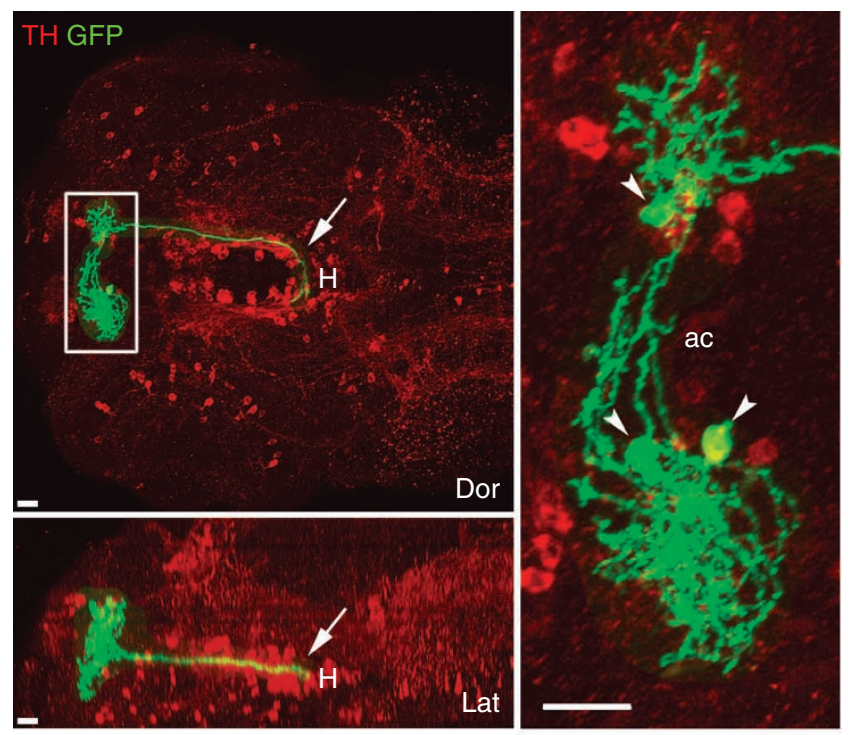

b

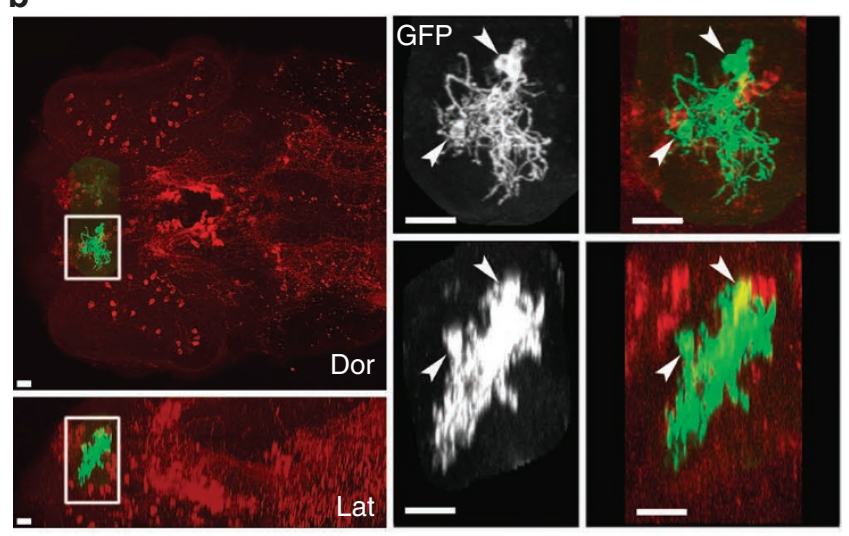

C

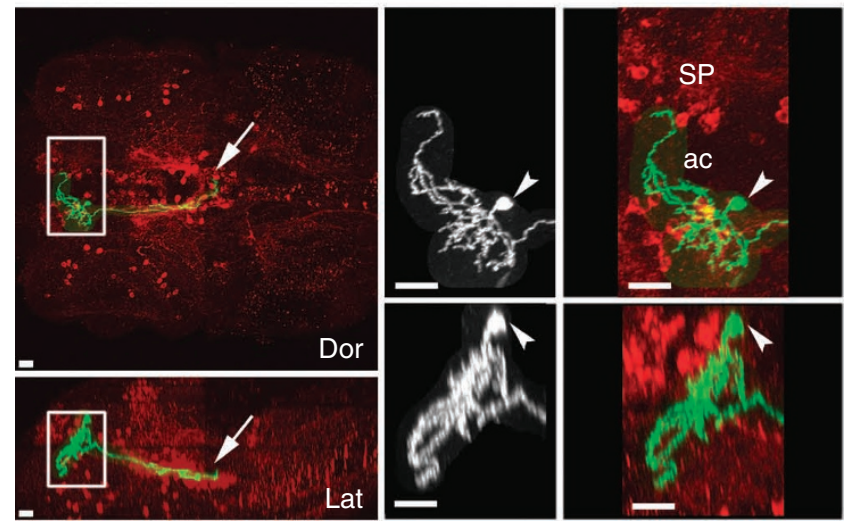

mood and cognition ${ }^{29}$. The highly conserved nature of the LC NA system and its postulated integrative role in setting basic states of the brain may inform an understanding of neurodegenerative diseases of the CA systems, including Parkinson's disease $\mathrm{e}^{30}$.

The detailed analysis of DA ascending and descending systems in the forebrain surprisingly revealed that prominent DA connectivity between the telencephalon and diencephalon is contributed both by descending projections of subpallial DA neurons and by ascending projections from the ventral diencephalon. The posterior tuberculum was previously shown to be a source of ascending DA projections into the SP in zebrafish ${ }^{11,12}$. We extend these findings

\section{Figure 8 | Extensive arbourization of subpallial DA neurons in the}

telencephalon. (a-c) Z projections of confocal stacks in dorsal (Dor) and lateral (Lat) views. (a) Three GFP-tagged subpallial neurons (arrowheads) reveal extensive arbourization within the subpallium. Subpallial neurons also frequently connect the left and right subpallium through the anterior commissure (ac; magnified in right panel). Furthermore, one of the subpallial DA neurons sends a descending projection into the hypothalamus (H; arrows). (b) Two subpallial DA neurons (arrowheads) form dense local arbours but no distant projections (magnified in middle and right panels). (c) A single subpallial soma (arrowheads) radiates dense local processes ventrally into telencephalic territories (magnified in middle and right panels), projects in the anterior commissure across the midline to the right subpallium (SP), and sends a descending axon into the hypothalamus (arrows). See Supplementary Movie 5. Anterior at left, dorsal at top; 4 d.p.f. larvae; anti-TH (red), anti-GFP (green; white in middle panels with black contrast). Scale bars, $20 \mu \mathrm{m}$.

by demonstrating that, at least by 4 d.p.f., Otp-dependent posterior tubercular DC2 and DC4 DA neurons are the only source of ascending DA activity. These ascending connections are surprisingly scarce in the larval brain, with on an average one or two ascending connections on each side of the brain. Individual Otp-dependent DA neurons with ascending DA projections also connect with other major axon branches to diencephalic, hindbrain and spinal cord targets. Our observations suggest that Otp-dependent DA neurons may potentially be the only DA system that integrates ascending and descending circuits contributing to motor control. They possibly serve a role in neuromodulatory integration of sensory function (pretectum, tectum, thalamus), neuroendocrine state and physiology (hypothalamus), motor control (hindbrain and spinal levels), as well as control of behaviour and cognition (SP). Mammalian Otp-specified A11 DA neurons ${ }^{15}$ have also been demonstrated to develop ascending neocortical and descending diencephalospinal projections $\mathrm{s}^{31-33}$, indicating that single-cell integration of rostral and caudal projections may be a component of an evolutionarily ancient DA neuromodulatory system. However, these studies showed neocortical/pallial but little or no subpallial A11 projections, raising the possibility that the final projection targets may not have been reached in 4-d.p.f. zebrafish larvae. Little is known about A11 function, although A11-lesioned rats are hyperactive ${ }^{34}$, and the system has been linked to Restless Legs Syndrome ${ }^{35}$ and descending command systems ${ }^{36}$.

Given that A11 DA neurons also express the hypothalamic marker Nkx2.1 (ref. 15), an early evolutionary origin of this system is possible. An anterior DA cell population that sends projections ventrally as well as descending into the spinal cord has been shown in Amphioxus, although a homology assignment to a brain region in vertebrates has not been possible ${ }^{37}$. In the protochordate Ciona intestinalis, dopamine cells localize to a hypothalamus-related domain of the sensory vesicle ${ }^{38}$. Diencephalospinal DA projections have also been described in agnathan vertebrates (lampreys) ${ }^{39}$. A11type DA neurons from fish to mammals are cospecified with neurosecretory hypothalamic neurons ${ }^{15,40,41}$, which also develop diencephalospinal projections. This has led to the hypothesis that the vasopressin/oxytocin systems may be involved in the spinal cord level control of sensorimotor, somatomotor and autonomic systems $s^{42,43}$. We therefore hypothesize that the Otp-dependent DA and neurosecretory systems may be the ancestral systems that synergistically set basic behavioural patterns such as active (fight/flight) or passive (freezing) avoidance.

Our data indicate that the previously proposed homology between the zebrafish posterior tubercular DA clusters and the mesodiencephalic DA groups A8-A10 residing in mammalian substantia nigra (SN) and ventral tegmental area ${ }^{44-46}$ needs to be reconsidered. Instead, the posterior tubercular DA groups should be considered 
A11 homologues. This homology is not only based on similarities in projection pattern, but also on conservation of transcription factors specifying these neurons ${ }^{15,41}$. Disputes about the transcription factor Nr4a2/Nurr1 controlling terminal differentiation in mice SN and ventral tegmental area DA neurons but not zebrafish posterior tubercular DA clusters ${ }^{44,47}$, further strengthen the notion that these clusters are different.

The numerous dense arbours of the subpallial DA neurons we observed within the SP indicate that a predominant telencephalic dopamine source in zebrafish may be derived locally. In all vertebrates, the striatum is one of the subdivisions of the $\mathrm{SP}^{48}$. In zebrafish, the striatum is a dorsal subdivision of the dorsal $\mathrm{SP}^{49}$. However, because of the eversion process of the telencephalon in teleostei, and the lack of specific molecular markers, the delimitation of the striatum within the zebrafish SP has not been identified. In contrast to the nigrostriatal contribution, dopamine input from intrinsic striatal dopamine neurons has received little attention in mammalian systems. Striatal DA neurons, confirmed as $\mathrm{TH}$ and DA transporter expressing small bipolar neurons ${ }^{50}$, have been described in both primates ${ }^{51}$ and rats $^{52}$. Morphologically, the extensive local subpallial DA arbours in zebrafish are reminiscent of cortical arbours derived from a single neuronal projection from the $\mathrm{SN}$ in the rodent midbrain ${ }^{53}$. In contrast, the ascending projections from otp-dependent posterior tubercular DC2/4 DA neurons do not arbourize in the larval SP, and thus do not broadly connect. Therefore, it is conceivable that the predominant DA input into the $\mathrm{SP}$ in zebrafish is derived from intrinsic DA neurons. Our findings that subsets of zebrafish subpallial DA neurons emanate contralateral subpallial projections and send descending projections into the caudal hypothalamus, indicate that these neurons may contribute to circuitries modulating neural functions in distant brain territories. Cell-based regenerative therapies have so far focused on midbrain A9-A10-type DA neurons or precursors, to be ectopically placed into the striatum ${ }^{54}$. Our description of an endogenous subpallial DA system should encourage research into sources for endogenous striatal DA neurons, which may potentially be more stable in the striatum than $\mathrm{SN}$-derived cells.

In contrast to the highly conserved hindbrain NA systems, major aspects of the far-projecting DA systems appear highly dynamic during evolution. Given overlapping target area profiles, we speculate that the NA and DA systems may have partially overlapping functions, as caudal hindbrain CA groups were reported to contain both NA and DA neurons in zebrafish and non-mammalian vertebrates $^{1,6}$. Although the NA systems might have remained stable during the teleost to tetrapod to mammalian transitions, the evolution of the distantly projecting DA systems may reflect the development of higher order control through the sequential addition of neuromodulatory mechanisms ${ }^{55}$. At an early stage, the local subpallial contribution may have been the predominant DA input into higher brain function. The increasing prominence of mesodiencephalic ascending DA projections may have facilitated integration of additional inputs that are, for example, derived from sensory systems. This includes the progressive involvement of the cortex/pallium in processing thalamic sensory information relayed through the basal ganglia of tetrapods ${ }^{56}$. Whether the mesodiencephalic ascending DA systems evolved by caudal expansion of ventral diencephalic DA groups ${ }^{56}$ or whether they evolved de novo in the context of mid-hindbrain organizer activity still needs to be resolved ${ }^{57}$.

\section{Methods}

Recombinant thrrasGFP BAC clones. We isolated a BAC clone (CHORB736O0777Q2) from the CHORI-211 zebrafish BAC library (http://bacpac.chori. org/zebrafish211.htm). Using primers pTARBAC2.1_F (5'-TTGCGGCCGCTAAT ACGACTCACTA-3') and pTARBAC2.1_1R (5'-ATTGAGGCACTTGGTATTTTTCGTT- $3^{\prime}$ ) for the ends of the BAC vector backbone, we sequenced both ends of the th insert to reveal that it contains $154 \mathrm{kbp}$ upstream and $12 \mathrm{kbp}$ transcribed region of the zebrafish tyrosine hydroxylase $(t h)$ gene (Supplementary Fig. S1). Two strategies were used to integrate ras membrane-anchored EGFP ( $m G F P)$ into the first exon of the th BAC clone. th:rasGFPsv (shuttle vector) was engineered using the R6K $\gamma$ RecA-based shuttle vector pLD53.SCA-E-B modified to contain a 1-kbp fragment $5^{\prime}$ of the th ATG and the mGFP reporter to be in-frame with the TH protein. Homologous integration into the BAC was verified by PCR (Supplementary Fig. S1). th:rasGFPsv was used in this study without removal of the vector sequence.

th:rasGFPvf (vector-free) was generated to reduce potential effects due to vector sequences, by using galK selection based recombineering ${ }^{58}$. Amplification of the galK selectable marker from $p G a l K$ was carried out using forward primer 5'-CCAGCGCTCCCAAGAGACCACAATATAGTTCCATCTGTTCCGCGCGCG CGCCATCCCTGTTGACAATTAATCATCGGCA-3' and reverse primer 5'-GTAA TAGAATCTGAGCGCTCGAGTTCAGACGCAGCTCTCCGGATGCTCTTTGTTC AGCACTGTCCTGCTCCTT-3' (th homology arms are in italics and underlined). The PCR product was electroporated into transformed bacteria and recombinants selected from $\mathrm{Gal}$ indicator plates were used in the second recombineering step. Forward primer 5'-CCAGCGCTCCCAAGAGACCACAATATAGTTCCATCTGTTC CGCGCGCGCGCCATCCCACCATGGTGAGCAAGGGC-3' and reverse primer 5'-GTAATAGAATCTGAGCGCTCGAGTTCAGACGCAGCTCTCCGGATGCTC TTTGTAAAAAACCTCCCACACCTCCCC-3' were used to amplify the targeting cassette from $m G F P$ (sequences are in bold) using proofreading Taq Polymerase (Advantage Taq, BD Clontech). Recombinants from Gal counter-selection plates were confirmed by PCR and sequencing. th:rasGFPvf was subsequently transformed from the SW102 Escherichia coli strain back into DH10B host cells. Double acetate precipitation and caesium chloride gradient separation were carried out to purify the modified BACs. DNA quality and concentration were checked by pulsed-field electrophoresis. Restriction digests, Southern hybridization, sequencing and PCR characterizations were carried out to verify single copy recombination of the reporter gene in the correct orientation (Supplementary Fig. S1). Comparing results of CA neuron labelling in embryos for both BAC constructs, we have observed no undesirable backbone effects for th:rasGFPsv, which contributed to $81.6 \%$ of our analyses.

Random GFP-labelling of th-expressing neurons. Zebrafish were maintained and bred under standard conditions at $28.5^{\circ} \mathrm{C}$. For microinjection into the cytoplasm of one-cell stage embryos, the modified BACs were prepared at $100-120 \mathrm{ng}^{-1}$ in $0.1 \mathrm{M}$ potassium chloride and $0.05 \%$ phenol red. To avoid melanin pigment formation, embryos were incubated in $0.2 \mathrm{mM}$ 1-Phenyl-2 thiourea (Sigma). At 3-4 d.p.f., embryos were mounted in 2.5\% methylcellulose containing Tricaine and screened for individual GFP-labelled neurons.

Immunolabelling and confocal microscopy. Immunofluorescence and immunohistochemistry were carried out as described ${ }^{12}$ using rabbit anti-tyrosine hydroxylase antibody (1:500 (ref. 15)) and chicken anti-GFP (1:400, Molecular Probes) or mouse anti-GFP antibodies (1:1,000, Roche). Alexa555- or Alexa594-conjugated goat anti-rabbit IgG (1:2,000 and 1:1,000, respectively, Molecular Probes) and Alexa488-conjugated goat anti-chicken IgG or anti-mouse IgG (1:600 and 1:1,000 respectively, Molecular Probes) were used as secondary antibodies. Embryos were mounted with $80 \%$ glycerol/1.2\% low-melting agarose/PBS.

Confocal $Z$ stacks were recorded with a Zeiss LSM 510 laser-scanning confocal microscope with pinhole diameters set to one airy unit for Alexa555 and Alexa488 labelled embryos using the 488- and 561-nm laser lines. Embryos stained with Alexa594 and Alexa488 were examined under Zeiss LSM 510 NLO with pinhole diameters between $360-520 \mu \mathrm{m}$ using the two-photon 770-nm laser line. All stacks were recorded with a $25 \times$ NA 0.8 multi-immersion lens (LD-LCI Plan-Apochromat) and glycerol immersion medium. Stack sizes are $1,024 \times 1,024$ pixel in $x y$ direction, with the number of $z$ planes depending on the thickness of the sample. The voxel sizes used were $0.5 \mu \mathrm{m} \times 0.5 \mu \mathrm{m} \times 1.3 \mu \mathrm{m}$ in the $z$ direction. Pixel times of $1.26-3.20 \mu$ s were used.

To better visualize neuronal projections in the context of the anatomical structures, differential interference contrast microscopy was used (Supplementary Fig. S9). Following confocal analysis, embryos of interest were incubated overnight in $1 \%$ goat serum/PBST containing DSB-X biotin-conjugated goat anti-chicken IgG (1:1,000, Molecular Probes), processed using biotinylated HRP and avidin reagents (Vectastain ABC HRP kit), and stained with the chromogen diaminobenzidine. Images were documented with DIC Axioscope2/AxioCam MRc (Zeiss) and processed with ImageJ (http://rsbweb.nih.gov/ij/).

Image processing and analysis. For far-projecting neurons, adjacent whole-brain image stacks were recorded. To enhance the visualization of GFP-tagged somata and their projections, we sought a simple solution to isolate them from nonspecific signals. In the whole-larvae preparations, immunofluorescence noise, auto-fluorescence (for example, some pigment cells), as well as other GFP expressing cells build up significant nonspecific signals when visualizations of image stacks are prepared. We wanted to avoid established noise-reduction approaches including thresholding or despeckling, given that fine CA processes in our stacks often had cross-section areas of very few pixels only. Therefore, we developed an image-processing pipeline for interactive in silico feature extraction, which is amenable to automated batch 
operation and includes a quality control element that allows independent and objective verification of 3D objects (Fig. 1).

The data set for a single brain recording was processed as follows (Fig. 1b): Maximum intensity projections (MIP) in dorsal and lateral directions and MIP movies of the revolving $3 \mathrm{D}$ brain image were created for quick visualization. The user proceeded to draw the dorsal and lateral segmentation masks over the MIPs using our ImageJ HDF5 plugin (http://lmb.informatik.uni-freiburg.de/lmbsoft/ imagej_plugins/). The 3D intersection $M$ of the $2 \mathrm{D}$ lateral $L$ and dorsal $D$ masks was computed as $M(x, y, z)=D(x, y) L(x, z)$, where $M, D$ and $L$ are scalar fields containing 1 for object pixels and 0 for background pixels. The resulting $3 \mathrm{D}$ mask was applied to the green channel, thereby allowing the segmentation of labelled neurons within a single brain and disregarding nonspecific signals and background (Fig. 1c-e). For quality control procedures as well as comparison to other projection tracking approaches, see Supplementary Methods and Fig. S10. All figures were assembled using Adobe Photoshop CS2 or Adobe Illustrator CS4. Brightness and contrast levels were adjusted in Photoshop to ensure that fine axonal and dendritic processes appear in print.

Identification and tracking of CA projections. Three-dimensional renderings of whole-brain image stacks were carefully analysed for the collection of complete information on individual neuron soma and projections.

Step 1: Identification of labelled CA neurons. On the basis of their anatomical position and correlation with anti-TH stained CA clusters, the neuronal CA subtype of the GFP-labelled CA neurons was identified. For each neuronal soma to be included in further analyses, the anti-TH (red) and anti-GFP (green) signals were analysed at high resolution and both colour channels were adjusted to verify the CA phenotype. For each neuron documented in the figures of this paper, the corresponding somata are displayed in Supplementary Fig. S11.

Step 2: Evaluation and classification of CA projections. Using the location of THir somata and axon tracts as anatomical reference, the projections of individual labelled neurons and their target areas were identified and classified. Histologically stained experimental whole-mount larvae preparations also enabled us to correlate projection paths and target areas with anatomical information (examples in Supplementary Figs S3 and S9).

Selectivity of th:rasGFP BAC labelled neurons. We assessed whether th:rasGFP BAC may drive ectopic expression of GFP in cells other than CA neurons. The embryos (9\%) analysed by confocal microscopy also showed anti-GFPir cells that did not correlate with anti-THir (data not shown). However, these cells appeared randomly distributed, including cells outside the CNS, such as somite cells. Such events may be explained by stochastic breaks of the BAC during integration and enhancer trap effects. The ectopic expression did not interfere with our analysis of CA neurons, which in each case could be clearly identified based on anti-THir.

Statistical analysis. To evaluate the likelihood that we may have missed significant projection patterns, we carried out statistical analysis. Given that different projection patterns for a specific neuron group may not be randomly formed and labelled, we analysed graphically how many projection patterns or target areas with a specific case number were identified for each DA group (Supplementary Tables S6, S7). Statistical analysis was carried out under the assumption that each pattern occurred at random, which is a simplification given that different groups had different cell numbers and differences in complexity of projection patterns. Binominal regression analysis was used to identify the interception with the $y$ axis. Plotting the number of times a specific projection pattern or target area was observed, revealed that we may have missed about $10 \%$ of potential target areas and about $20 \%$ of projection types. However, the several-fold increase in number of analysed embryos required to reduce the zero class significantly was not technically feasible in this study.

A non-random effect on the level of saturation would hypothetically be possible if the th:rasGFP BAC has a bias in labelling only a subset of DA neurons. However, we observed that each group contributes to the total number of GFP-labelled cells at approximately the same ratio as its THir cell count compared with the total number of THir cells. Therefore, we believe that we have surveyed the complete set of THir cell types in the larvae. However, we cannot fully exclude the underrepresentation of a specific THir cell type.

\section{References}

1. Smeets, W. J. A. J. \& González, A. Catecholamine systems in the brain of vertebrates: new perspectives through a comparative approach. Brain Res. Brain Res. Rev. 33, 308-379 (2000).

2. Smeets, W. J. A. J. \& Reiner, A Phylogeny and Development of Catecholamine Systems in the CNS of Vertebrates (Cambridge University Press, 1994).

3. Budick, S. A. \& O'Malley, D. M. Locomotor repertoire of the larval zebrafish: swimming, turning and prey capture. J. Exp. Biol. 203, 2565-2579 (2000).

4. Rink, E. \& Wullimann, M. F. Development of the catecholaminergic system in the early zebrafish brain: an immunohistochemical study. Brain Res. Dev. Brain Res. 137, 89-100 (2002).

5. Kaslin, J. \& Panula, P. Comparative anatomy of the histaminergic and other aminergic systems in zebrafish (Danio rerio). J. Comp. Neurol. 440, 342-377 (2001).
6. McLean, D. L. \& Fetcho, J. R. Ontogeny and innervation patterns of dopaminergic, noradrenergic, and serotonergic neurons in larval zebrafish. J. Comp. Neurol. 480, 38-56 (2004).

7. Holzschuh, J., Ryu, S., Aberger, F. \& Driever, W. Dopamine transporter expression distinguishes dopaminergic neurons from other catecholaminergic neurons in the developing zebrafish embryo. Mech. Dev. 101, 237-243 (2001).

8. Chen, Y. C., Priyadarshini, M. \& Panula, P. Complementary developmental expression of the two tyrosine hydroxylase transcripts in zebrafish. Histochem. Cell Biol. 132, 375-381 (2009).

9. Filippi, A., Mahler, J., Schweitzer, J. \& Driever, W. Expression of the paralogous tyrosine hydroxylase encoding genes th 1 and th 2 reveals the full complement of dopaminergic and noradrenergic neurons in zebrafish larval and juvenile brain. J. Comp. Neurol. 518, 423-438 (2010).

10. Yamamoto, K., Ruuskanen, J. O., Wullimann, M. F. \& Vernier, P. Two tyrosine hydroxylase genes in vertebrates new dopaminergic territories revealed in the zebrafish brain. Mol. Cell Neurosci. 43, 394-402 (2010).

11. Rink, E. \& Wullimann, M. F. The teleostean (zebrafish) dopaminergic system ascending to the subpallium (striatum) is located in the basal diencephalon (posterior tuberculum). Brain Res. 889, 316-330 (2001)

12. Kastenhuber, E., Kratochwil, C. F., Ryu, S., Schweitzer, J. \& Driever, W. Genetic dissection of dopaminergic and noradrenergic contributions to catecholaminergic tracts in early larval zebrafish. J. Comp. Neurol. 518, 439-458 (2010).

13. Blechman, J. et al. Specification of hypothalamic neurons by dual regulation of the homeodomain protein Orthopedia. Development 134, 4417-4426 (2007).

14. Del Giacco, L. et al. Differential regulation of the zebrafish orthopedia 1 gene during fate determination of diencephalic neurons. BMC Dev. Biol. 6, 50 (2006).

15. Ryu, S. et al. Orthopedia homeodomain protein is essential for diencephalic dopaminergic neuron development. Curr. Biol. 17, 873-880 (2007).

16. Ma, P. M. Catecholaminergic systems in the zebrafish. II. Projection pathways and pattern of termination of the locus coeruleus. J. Comp. Neurol. 344, 256-269 (1994)

17. Ma, P. M. Catecholaminergic systems in the zebrafish. I. Number, morphology, and histochemical characteristics of neurons in the locus coeruleus. J. Comp. Neurol. 344, 242-255 (1994).

18. Ma, P. M. Catecholaminergic systems in the zebrafish. III. Organization and projection pattern of medullary dopaminergic and noradrenergic neurons. J. Comp. Neurol. 381, 411-427 (1997).

19. Sallinen, V. et al. MPTP and MPP+ target specific aminergic cell populations in larval zebrafish. I. Neurochem. 108, 719-731 (2009).

20. Ma, P. M. Catecholaminergic systems in the zebrafish. IV. Organization and projection pattern of dopaminergic neurons in the diencephalon. J. Comp. Neurol. 460, 13-37 (2003).

21. Kasthuri, N. \& Lichtman, J. W. The rise of the 'projectome'. Nat. Methods 4, 307-308 (2007).

22. Ma, P. M. \& Lopez, M. Consistency in the number of dopaminergic paraventricular organ-accompanying neurons in the posterior tuberculum of the zebrafish brain. Brain Res. 967, 267-272 (2003).

23. Lu, J., Tapia, J. C., White, O. L., Lichtman, J. W. \& Bargmann, C. I. The interscutularis muscle connectome. PLoS Biol. 7, e32 (2009).

24. White, J., Southgate, E., Thomson, J. \& Brenner, S. The structure of the nervous system of the nematode Caenorhabditis elegans. Philos. Transact. B 314, 1 (1986)

25. Moore, R. Y. \& Bloom, F. E. Central catecholamine neuron systems: anatomy and physiology of the norepinephrine and epinephrine systems. Annu. Rev. Neurosci. 2, 113-168 (1979).

26. Northcutt, R. G. Connections of the lateral and medial divisions of the goldfish telencephalic pallium. J. Comp. Neurol. 494, 903-943 (2006).

27. Rink, E. \& Wullimann, M. F. Connections of the ventral telencephalon (subpallium) in the zebrafish (Danio rerio). Brain Res. 1011, 206-220 (2004).

28. Yoshimoto, M. \& Yamamoto, N. Ascending general visceral sensory pathways from the brainstem to the forebrain in a cichlid fish, Oreochromis (Tilapia) niloticus. J. Comp. Neurol. 518, 3570-3603 (2010).

29. Sara, S. J. The locus coeruleus and noradrenergic modulation of cognition. Nat. Rev. Neurosci. 10, 211-223 (2009).

30. Rommelfanger, K. S. \& Weinshenker, D. Norepinephrine: the redheaded stepchild of Parkinson's disease. Biochem. Pharmacol. 74, 177-190 (2007).

31. Takada, M., Li, Z. K. \& Hattori, T. Single thalamic dopaminergic neurons project to both the neocortex and spinal cord. Brain Res. 455, 346-352 (1988).

32. Takada, M. Widespread dopaminergic projections of the subparafascicular thalamic nucleus in the rat. Brain Res. Bull. 32, 301 (1993).

33. Björklund, A. \& Skagerberg, G. Evidence for a major spinal cord projection from the diencephalic A11 dopamine cell group in the rat using transmitterspecific fluorescent retrograde tracing. Brain Res. 177, 170-175 (1979).

34. Qu, S. et al. Locomotion is increased in al1-lesioned mice with iron deprivation: a possible animal model for restless legs syndrome. J. Neuropathol. Exp. Neurol. 66, 383-388 (2007).

35. Clemens, S., Rye, D. \& Hochman, S. Restless legs syndrome: revisiting the dopamine hypothesis from the spinal cord perspective. Neurology 67, 125-130 (2006). 
36. Jordan, L. M., Liu, J., Hedlund, P. B., Akay, T. \& Pearson, K. G. Descending command systems for the initiation of locomotion in mammals. Brain Res. Rev. 57, 183-191 (2008).

37. Moret, F., Guilland, J. C., Coudouel, S., Rochette, L. \& Vernier, P. Distribution of tyrosine hydroxylase, dopamine, and serotonin in the central nervous system of amphioxus (Branchiostoma lanceolatum): implications for the evolution of catecholamine systems in vertebrates. J. Comp. Neurol. 468, 135-150 (2004).

38. Moret, F. et al. The dopamine-synthesizing cells in the swimming larva of the tunicate Ciona intestinalis are located only in the hypothalamus-related domain of the sensory vesicle. Eur. J. Neurosci. 21, 3043-3055 (2005).

39. Barreiro-Iglesias, A., Villar-Cervino, V., Anadon, R. \& Rodicio, M. Descending brain-spinal cord projections in a primitive vertebrate, the lamprey: cerebrospinal fluid-contacting and dopaminergic neurons. J. Comp. Neurol. 511 (2008).

40. Acampora, D. et al. Progressive impairment of developing neuroendocrine cel lineages in the hypothalamus of mice lacking the Orthopedia gene. Genes Dev. 13, 2787-2800 (1999).

41. Lohr, H., Ryu, S. \& Driever, W. Zebrafish diencephalic A11-related dopaminergic neurons share a conserved transcriptional network with neuroendocrine cell lineages. Development 136, 1007-1017 (2009).

42. Rose, J. D. \& Moore, F. L. Behavioral neuroendocrinology of vasotocin and vasopressin and the sensorimotor processing hypothesis. Front Neuroendocrinol. 23, 317-341 (2002).

43. Kerman, I. A. Organization of brain somatomotor-sympathetic circuits. Exp. Brain Res. 187, 1-16 (2008).

44. Blin, M., Norton, W., Bally-Cuif, L. \& Vernier, P. NR4A2 controls the differentiation of selective dopaminergic nuclei in the zebrafish brain. Mol. Cell. Neurosci. 39, 592-604 (2008)

45. Kapsimali, M., Bourrat, F. \& Vernier, P. Distribution of the orphan nuclear receptor Nurr1 in medaka (Oryzias latipes): cues to the definition of homologous cell groups in the vertebrate brain. J. Comp. Neurol. 431, 276-292 (2001).

46. Rink, E. \& Wullimann, M. F. Connections of the ventral telencephalon and tyrosine hydroxylase distribution in the zebrafish brain (Danio rerio) lead to identification of an ascending dopaminergic system in a teleost. Brain Res. Bull. 57, 385-387 (2002).

47. Filippi, A. et al. Expression and function of nr4a2, $\operatorname{lm} x 1 \mathrm{~b}$, and pitx3 in zebrafish dopaminergic and noradrenergic neuronal development. BMC Dev. Biol. 7, 135 (2007).

48. Northcutt, R. G. Evolution of the telencephalon in nonmammals. Ann. Rev. Neurosci. 4, 301-350 (1981).

49. Mueller, T., Wullimann, M. F. \& Guo, S. Early teleostean basal ganglia development visualized by zebrafish Dlx2a, Lhx6, Lhx7, Tbr2 (eomesa), and GAD67 gene expression. J. Comp. Neurol. 507, 1245-1257 (2008).

50. Betarbet, R. et al. Dopaminergic neurons intrinsic to the primate striatum. J. Neurosci. 17, 6761-6768 (1997).

51. Dubach, M. et al. Primate neostriatal neurons containing tyrosine hydroxylase: immunohistochemical evidence. Neurosci. Lett. 75, 205-210 (1987).

52. Tashiro, Y. et al. Tyrosine hydroxylase-like immunoreactive neurons in the striatum of the rat. Neurosci. Lett. 97, 6-10 (1989).
53. Matsuda, W. et al. Single nigrostriatal dopaminergic neurons form widely spread and highly dense axonal arborizations in the neostriatum. J. Neurosci. 29, 444-453 (2009).

54. Kim, J. et al. Dopamine neurons derived from embryonic stem cells function in an animal model of Parkinson's disease. Nature 418, 50-56 (2002).

55. Katz, P. S. Evolution and development of neural circuits in invertebrates. Curr. Opin. Neurobiol. 17, 59-64 (2007)

56. Smeets, W., Marin, O. \& Gonzales, A. Evolution of the basal ganglia: new perspectives through a comparative approach. J. Anat. 196, 501-517 (2000).

57. Prakash, N. \& Wurst, W. Development of dopaminergic neurons in the mammalian brain. Cell Mol. Life Sci. 63, 187-206 (2006).

58. Warming, S., Costantino, N., Court, D. L., Jenkins, N. A. \& Copeland, N. G. Simple and highly efficient BAC recombineering using galK selection. Nucleic Acids Res. 33, e36 (2005).

\section{Acknowledgments}

We thank S. Götter for excellent fish care, and N. Kirstein for technical support. Special thanks to the staff of the ZBSA Life Imaging Center for excellent confocal microscopy resources. We thank D. Onichtchouk, A. Filippi and the staff of Bitplane AG for technica discussion. We are grateful to G. Nikkhah, T. Müller, J. Schweitzer, M. Jaenicke, H. Löhr, J. Holzschuh, M. Spelleken and E. Beddows for suggestions on the manuscript. This work was funded by the Deutsche Forschungsgemeinschaft DFG Grants SFB505-B7, SFB780-B6, SFB592-Z2, Research Training Group 1104 and Excellence Initiative of the German Federal and State Governments (EXC 294), as well as by the EC program mesDANEURODEV.

\section{Author contributions}

W.D. and T.L.T. conceived and designed the experiments. S.R. and T.L.T. constructed the engineered BAC clones. T.L.T. conducted the projection mapping experiments and collected all data. R.N. contributed to confocal imaging. T.L.T., O.R. and W.D. analysed data. T.L.T., O.R. and W.D. wrote the paper.

\section{Additional information}

Supplementary Information accompanies this paper at http://www.nature.com/ naturecommunications

Competing financial interests: The authors declare no competing financial interests.

Reprints and permission information is available online at http://npg.nature.com/ reprintsandpermissions/

How to cite this article: Tay, T. L. et al. Comprehensive catecholaminergic projectome analysis reveals single neuron integration of zebrafish ascending and descending dopaminergic systems. Nat. Commun. 2:271 doi: 10.1038/ncomms1171 (2011).

License: This work is licensed under a Creative Commons Attribution-NonCommercialNoDerivative Works 3.0 Unported License. To view a copy of this license, visit http:// creativecommons.org/licenses/by-nc-nd/3.0/ 